\title{
Purity monitoring in medical gas supply lines with quantum cascade laser technology
}

\author{
Henrik Zimmermann ${ }^{1}$, Mathias Wiese ${ }^{1}$, Luigi Fiorani ${ }^{2}$, and Alessandro Ragnoni ${ }^{2}$ \\ ${ }^{1}$ neoplas control GmbH, Walther-Rathenau-Str. 49a, 17489 Greifswald, Germany \\ ${ }^{2}$ Loccioni Group, Via Fiume 16, 60030 Angeli di Rosora, Ancona, Italy
}

Correspondence to: Henrik Zimmermann (zimmermann@ neoplas-control.de)

Received: 30 September 2016 - Revised: 18 March 2017 - Accepted: 27 March 2017 - Published: 13 April 2017

\begin{abstract}
Because of their direct impact on patients, medical supply lines are under strict regulations and have to be monitored in terms of purity on a regular basis. State-of-the-art measurement solutions do not allow for continuous bedside monitoring. The aim of the presented project is to provide a compact multispecies monitoring system based on the latest quantum cascade laser technologies.
\end{abstract}

\section{Introduction}

Medical gas supply lines in hospitals are subject to strict regulations. Besides the establishment of modern management techniques, continuous monitoring of the distribution lines could avoid the fatal accidents that unfortunately still happen to patients, especially after maintenance is performed on older buildings (Weller et al., 2007; Cornwell et al., 2016). Concentration limits are defined for every gas line by local or national regulations, such as the European Pharmacopeia. The most important impurities are $\mathrm{CO}_{2}, \mathrm{CO}, \mathrm{H}_{2} \mathrm{~S}, \mathrm{SO}_{2}, \mathrm{NO}_{x}$, $\mathrm{H}_{2} \mathrm{O}, \mathrm{O}_{2}, \mathrm{~N}_{2} \mathrm{O}$ and oil. Currently, monitors can be found on the market that are either compact, user-friendly mono-gas systems used for discrete low-accuracy inspections or multigas, bulky and quite expensive instrumentation developed to perform periodic checks on gas access; however, these definitely do not allow for comfortable bedside operation. A compact, cost-effective multi-gas sensing technology would ensure higher safety potential as it could be installed directly at the sampling point to perform distributed continuous monitoring.

In this context, sensors based on quantum cascade laser technology, which have proven their applicability in demanding conditions (Anscombe, 2011) spanning from the environmental monitoring sector to process monitoring and control applications (Röpcke et al., 2012), may offer a suitable solution.
The present study introduces a new approach to the bedside purity monitoring of medical gases in hospitals. The three main supply lines to check are the oxygen line, the nitrogen protoxide line and the compressed air line.

As a first step, a single-species monitor focusing on $\mathrm{SO}_{2}$ detection is presented (Q-MACS Trace), which is the milestone for the multispecies solution based on the latest technology developments (http://www.mirifisens-project.eu).

\section{Experimental setup}

Two sketches, one for the sensor assembly and the other for the optical subsystem of the Q-MACS Trace compact sensor configuration, are presented in Fig. 1.

As a laser absorption spectrometer based on the direct absorption technique, the Q-MACS Trace compact $\mathrm{SO}_{2}$ sensor acquires the transmission signal with an infrared detector after passing a measurement cell of Herriott type with a volume of $0.5 \mathrm{~L}$ which provides an absorption path of $0.5 \mathrm{~L}$. A portion of the laser beam, which is reflected at the coupling window of the measurement cell, is guided through a gas cuvette with a fixed filling and collected by a second detector. The absorption feature from the cuvette is used to stabilize the spectral position of the laser by means of temperature tuning. To address a suitable $\mathrm{SO}_{2}$ absorption feature while taking into account possible cross sensitivities, a QCL emitting around $7.4 \mu \mathrm{m}$ is applied as a narrow-band light source providing an optical power of about $10 \mathrm{~mW}$. The laser repet- 

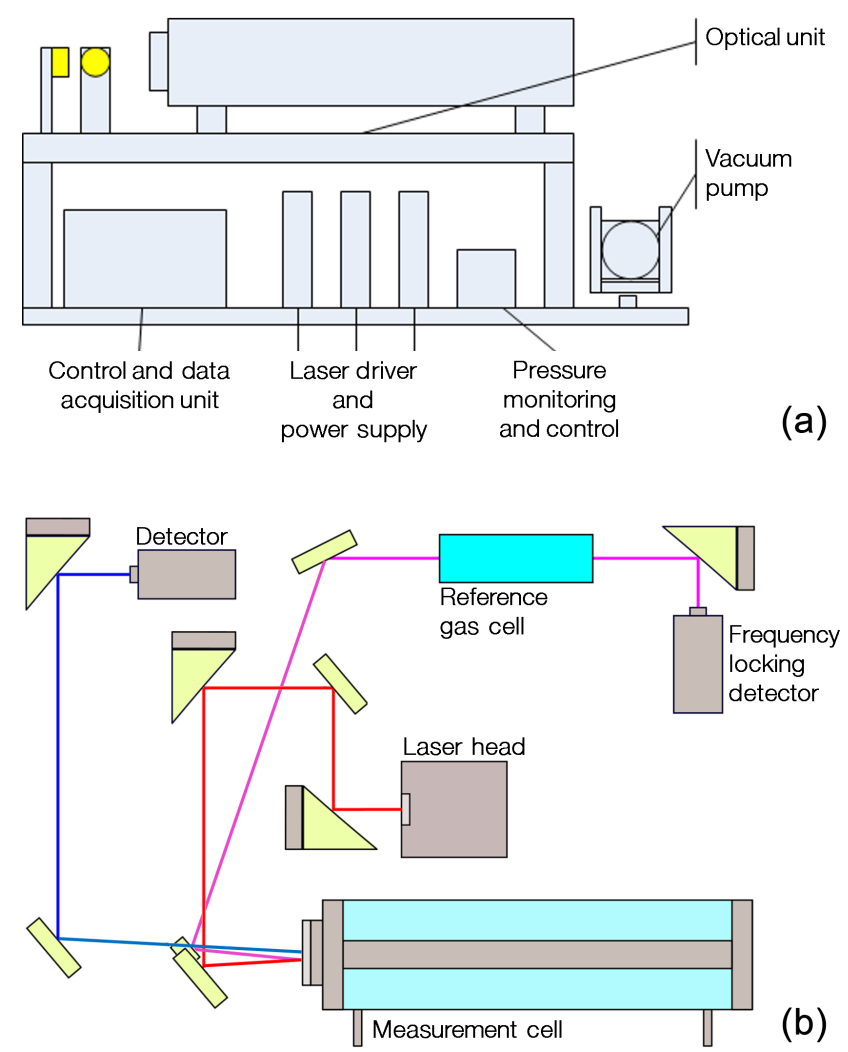

Figure 1. The general sensor assembly with electronics, optics (a) and the detailed optical sensor setup (b).

itively tunes over a spectral range of $0.7 \mathrm{~cm}^{-1}$ via a monotonic current ramp with a repetition rate of $2 \mathrm{kHz}$. To reduce the heat impact of the laser and allow it to reliably achieve the laser temperature of $7^{\circ} \mathrm{C}$ via Peltier cooling, the intermittent scanning regime introduced by Fischer is applied (Fischer et al., 2014). A duty cycle of $20 \%$ is selected. This driving scheme allows for the acquisition of a complete $\mathrm{SO}_{2}$ absorption feature with very high resolution. In Fig. 2, the processed portion of $0.2 \mathrm{~cm}^{-1}$ from the acquired detector signal and the corresponding fit result are shown. This signal is taken at the chosen full-scale value of $7 \mathrm{ppm}$ at $60 \mathrm{mbar}$ and a $56 \mathrm{~m}$ absorption path, matching an absolute density of $1.03 e+13$ molecules $\mathrm{cm}^{-3}$.

The acquired signal is analyzed with respect to the wellknown molecular parameters by means of the LevenbergMarquardt algorithm, which ensures nearly calibration-free measurements. In terms of the introduced $\mathrm{SO}_{2}$ measurements, these parameters are taken from the HITRAN molecular absorption database (Rothman et al., 2013).

The gas dilution system consisted of two units (MF1; MKS Instruments, Inc., Andover, MA, USA) of mass flow controllers and a diaphragm pump (Thomas 7011Z DC; Gardner Denver, Milwaukee, WI, USA). One mass flow controller provides a range of 0 to $200 \mathrm{sccm}$ and another one comes with a range of 0 to $10 \mathrm{slm}$. This set of flow con-

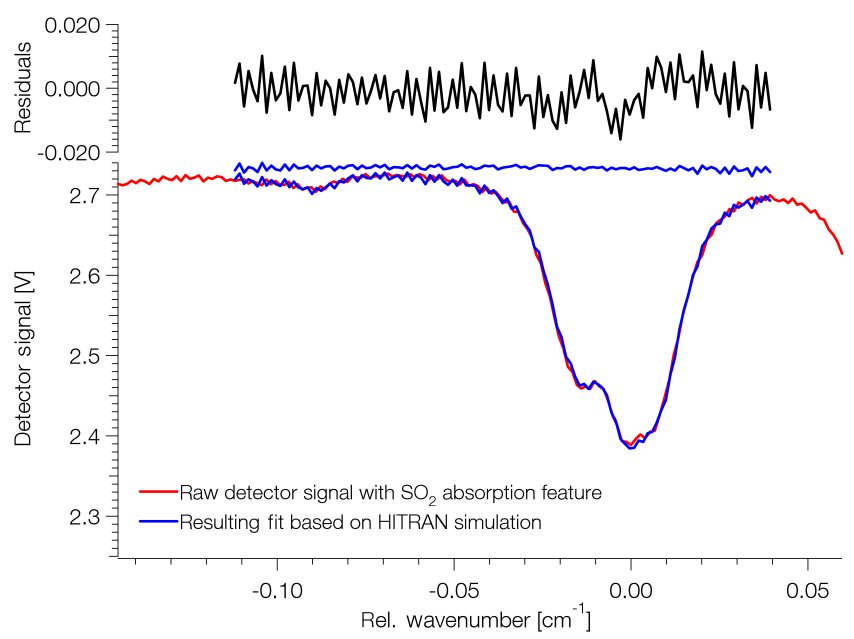

Figure 2. The acquired detector signal with the $\mathrm{SO}_{2}$ absorption feature corresponding to $7 \mathrm{ppm}$ at $60 \mathrm{mbar}$ and the fit result.

Table 1. A list of the dilution steps, the resulting $\mathrm{SO}_{2}$ mixing ratios and the achievable accuracy.

\begin{tabular}{lrrr}
\hline $\begin{array}{l}\text { Target } \\
(\mathrm{ppb})\end{array}$ & $\begin{array}{r}\mathrm{SO}_{2} \\
(\mathrm{sccm})\end{array}$ & $\begin{array}{r}\mathrm{N}_{2} \\
(\mathrm{sccm})\end{array}$ & $\begin{array}{r}\text { Accuracy } \\
(\mathrm{ppb})\end{array}$ \\
\hline 0 & 0 & 300 & $+15 /-0$ \\
70 & 2 & 298 & $+23 /-19$ \\
170 & 5 & 295 & $+35 /-30$ \\
350 & 10 & 290 & $+55 /-47$ \\
690 & 20 & 280 & $+94 /-80$ \\
1700 & 50 & 250 & $+209 /-180$ \\
3500 & 100 & 200 & $+395 /-344$ \\
5200 & 150 & 150 & $+574 /-502$ \\
7000 & 200 & 100 & $+747 /-655$ \\
\hline
\end{tabular}

trollers allows for reasonable accuracy in the dilution of gas standards over a very wide range of dilution ratios with the selection of the appropriate settings on the mass flow controllers. The distinct concentration steps, which were alternatingly tuned as a continuous flow through the measurement cell, are reflected in Table 1.

This dilution system provides the test atmospheres for the analyzers that are undergoing testing.

The pressure in the sample cell is monitored via an HPS Series 902 piezo transducer (MKS Instruments, Inc., Andover, MA, USA), which provides a range of application of 0 to 1300 mbar. Using this pressure gauge, the pressure in the cell was maintained at a fixed value of $\sim 60$ mbar during the tests. No active pressure control was applied during this test.

\section{Statistical methods}

The statistical methods used to evaluate the quantitative performance factors are presented in this chapter. Since no alternative concept for the detection of $\mathrm{SO}_{2}$ was available dur- 
ing the test, the evaluation of the performance parameters for the sensor had to be based on the calculated mixing ratios, which depend on the settled flow rates. This approach limits the types of statistical comparisons that could be applied. Qualitative observations were also used to evaluate the verification test data.

The linearity factor is assessed by linear regression with the calibration concentration as the independent variable and the analyzer response as the dependent variable. The calibration model is given in Eq. (1):

$Y_{C}=h(c)+\operatorname{error}_{C}$,

where $Y_{C}$ is the analyzer response to a challenge concentration $c, h(c)$ is a linear calibration curve and the error term is assumed to be normally distributed. The variability $\sigma$ in the measured concentration values $c$ was modeled by the relationship expressed in the following equation:

$\sigma_{C}^{2}=\alpha+k c^{\beta}$

where $\alpha, k$ and $\beta$ are constants to be estimated from the data. After determining the relationship between the mean and the variability, the appropriate weighting is determined by Eq. (3):

$w_{C}=\frac{1}{\sigma_{C}^{2}}$.

The form of the regression model to be fitted is expressed in Eq. (4):

$c=h^{-1}\left(Y_{C}\right)=\frac{\left(Y_{C}-\alpha_{0}\right)}{\alpha_{1}}$.

The concentration values were calculated from the estimated calibration curve using the following formula:

$h(c)=\alpha_{0}+\alpha_{1} c$.

A test for the departure from linearity is carried out by comparing the residual sum of squares to a chi-square distribution with $6-2=4$ degrees of freedom, as given with Eq. (6):

$\sum_{i=1}^{6}\left(\bar{Y} c_{i}-\alpha_{0}-\alpha_{1} c_{i}\right)^{2} n_{C_{i}} w_{C_{i}}$

where $n_{C}$ is the number of replicates at concentration $c$.

The response time of the analyzers to a step change in the analyte concentration was calculated by determining the total change in the response due to the step change (either an increase or a decrease) in concentration, and then determining the point in time at which $95 \%$ of that change was achieved. Both the rise and fall times were determined. Using data taken at intervals of $1 \mathrm{~s}$, the calculation is carried out by Eq. (7):

$R_{\text {Total }}=R_{\mathrm{a}}-R_{\mathrm{b}}$,

where $R_{\mathrm{a}}$ is the final response of the analyzer to the test gas after the step change, and $R_{\mathrm{b}}$ is the final response of the analyzer before the step change. The analyzer response that indicates the response time is then calculated by applying Eq. (8):

$R=0.95 R_{\text {Total }}$

The point in time at which this response occurs is determined by inspecting the response time data, and the response time is calculated according to Eq. (9):

Time $_{\text {Response }}=$ Time $_{95} \%-$ Time $_{I}$,

where Time $95 \%$ is the time at which $R$ occurs, and Time ${ }_{I}$ is the time at which the step change in concentration was imposed. Since only one determination was made, the precision of the rise and fall time results could not be estimated.

The detection limit (LOD) was defined as the smallest true concentration at which the analyzers expected the response to exceed the calibration curve at zero concentration by 3 times the standard deviation of the analyzer zero reading. The LOD is then determined by applying Eq. (10):

$\mathrm{LOD}=\frac{\left[\left(\alpha_{0}+3 \sigma_{0}\right)-\alpha_{0}\right]}{\alpha_{1}}=\frac{3 \sigma_{0}}{\alpha_{1}}$

Here, $\sigma_{0}$ is the estimated standard deviation at zero concentration. Note that the validity of the detection limit estimate and its standard error depend on the validity of the assumption that the fitted linear calibration model accurately represents the response down to zero concentration.

The statistical procedures for assessing the zero and span drift were similar to those used to assess the interrupted sampling. The zero (span) drift was calculated as the arithmetic difference between the zero and span values respectively obtained before and after the sampling of the source emissions. During this test, no estimate of the precision of the zero and span drift values was made.

\section{Results and discussion}

A Q-MACS Trace analyzer prototype was tested for the most highly sensitive online monitoring of $\mathrm{SO}_{2}$ traces. The laboratory tests were designed to challenge the analyzer over its 


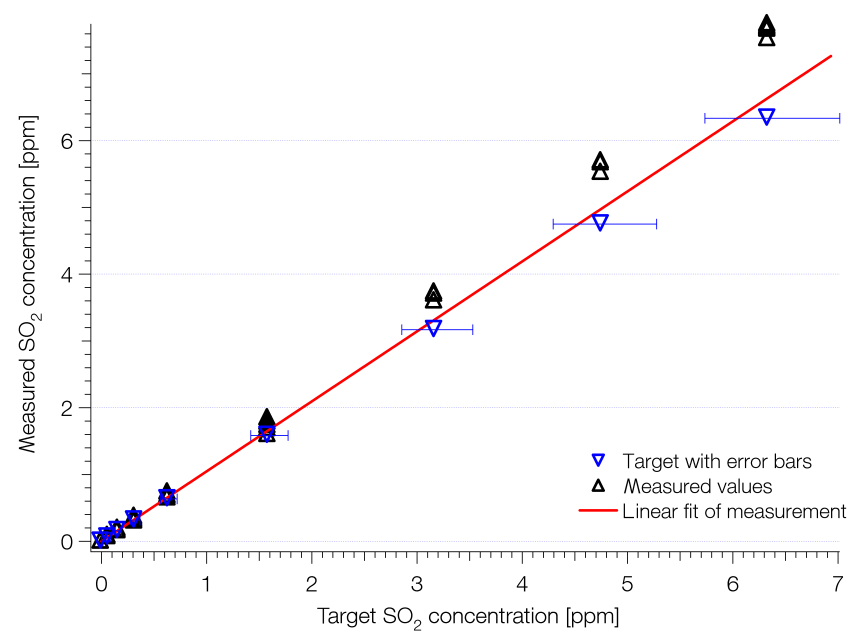

Figure 3. The linearity results for the Q-MACS Trace setup with a $1 \mathrm{~s}$ acquisition time.

full range under a variety of conditions. These tests were performed using certified standard gases and a gas dilution system. The gas standards were diluted with high-purity gases to produce the desired range of concentrations with known accuracy. The laboratory testing was conducted primarily by supplying known gas mixtures to the Q-MACS Trace analyzer from the gas delivery system. The linearity of the response of the Q-MACS Trace analyzer was tested with 30point calibrations of the $\mathrm{SO}_{2}$ gas filling. Prior to this check, the analyzer is provided with the appropriate zero gas $\left(\mathrm{N}_{2}\right)$ and then with a span gas concentration of $7 \mathrm{ppm}$ of $\mathrm{SO}_{2}$, which is defined in this verification test to be the nominal range of the analyzer. After any necessary adjustments to the analyzer to match that span value, the 30 -point check proceeded without further adjustments. The 30 points consisted of three replicates each at 70,170, 350 and $690 \mathrm{ppb}$ and $1.7,3.5,5.2$ and $7 \mathrm{ppm}$ in random order interspersed with six replicates of zero gas. Following the completion of all 30 points, the 0 and $100 \%$ spans were repeated without adjustment of the analyzer. The zero and span drift will be evaluated using the data generated in the linearity and the accuracy tests. The zero and span drift is determined as the difference in the response to the zero and span gases in these two tests. This comparison will be made for all zero and span responses using data from the linearity and the accuracy tests. Figure 3 shows the linearity results obtained from the linearity tests for the Q-MACS Trace analyzer, which was configured for the $\mathrm{SO}_{2}$ measurements.

In Table 2, the linear equations for the system configuration developed from these data are shown.

The quite low value for the regression coefficient in the linearity tests is caused by the rather high uncertainty that the chosen dilution system provides for the $\mathrm{N}_{2}$ dilution gas at lower flow rates. The uncertainties in the dilution system are listed in Table 1 and visualized by the error bars in Fig. 3.
Table 2. The statistical results of the linearity test.

\begin{tabular}{rrr}
\hline Intercept (ppb) & Slope & $R^{2}$ \\
\hline$-0.271 \pm 3.74$ & $1.048 \pm 0.02$ & 0.823 \\
\hline
\end{tabular}

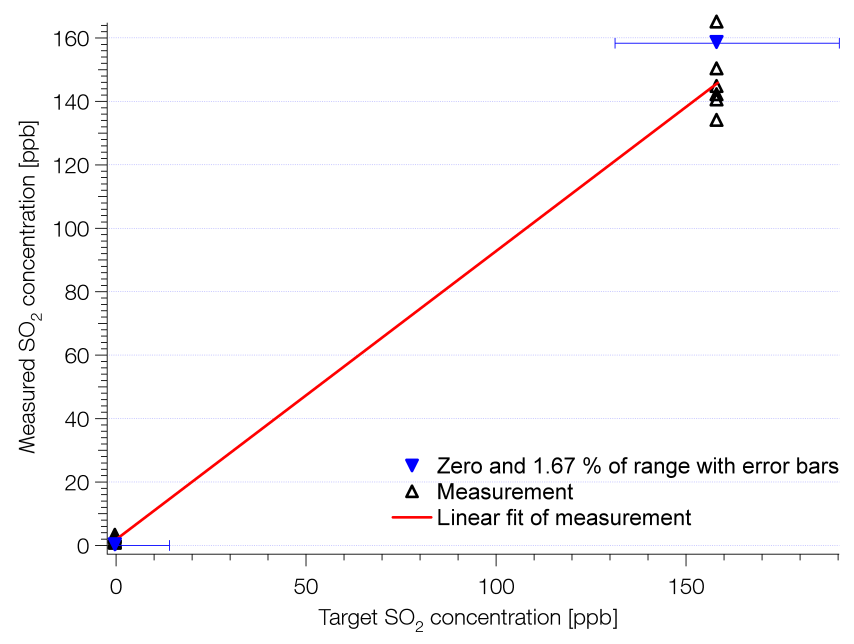

Figure 4. The results of the linear fit for the measured values at 0 and $1.67 \%$ of the range.

For the linear fit, the concentration values are weighted with the uncertainties of the dilution system to respect the deviation of the finally settled concentrations from the targeted ones.

Table 3 shows the detection limits for each configuration of the Q-MACS Trace analyzer tested, determined from the detection limit procedure described in the previous section. Figure 4 visualizes the results of the linear fit valid for 0 to $1.67 \%$ of the range. The calculated detection limit for $1 \mathrm{~s}$ of acquisition time is $22.99 \mathrm{ppb}$, corresponding to a density of $3.36 e+10$ molecules $\mathrm{cm}^{-3}$.

The response time for the sensor based on a step change in the analyte concentration was determined to about $14 \pm 1 \mathrm{~s}$ with the acquisition time limited to $1 \mathrm{~s}$.

Figure 5 shows the trend in the $\mathrm{SO}_{2}$ sensor signal while the $\mathrm{SO}_{2}$ concentration in the gas stream is changed between 0 and $7 \mathrm{ppm}$ (span) for seven independent measurements. The decrease in the concentration level after about $20 \mathrm{~s}$ is due to the control behavior of the chosen mass flow controller.

The zero and span data taken at the start and end of the linearity test are shown in Table 4.

The observed drift values are shown in Table 5 as the differences between the pretest and the posttest concentration measurements. Furthermore, Table 5 also presents the zero and span drifts as a percent of the span gas concentrations. The zero drift for the tested sensor was less than $0.04 \%$ of the respective span gas concentration. The span drift was less than $0.22 \%$ of the respective span concentration. 
Table 3. The detection limit determined according to the $\mathrm{SO}_{2}$ measurements.

\begin{tabular}{lr}
\hline Slope & $0.969 \pm 0.086$ \\
Standard deviation & 4.26 \\
for zero values $(\mathrm{ppb})$ & \\
Average noise $(\mathrm{ppb})$ & 7.67 \\
Detection limit $(\mathrm{ppb})$ & 22.99 \\
\hline
\end{tabular}

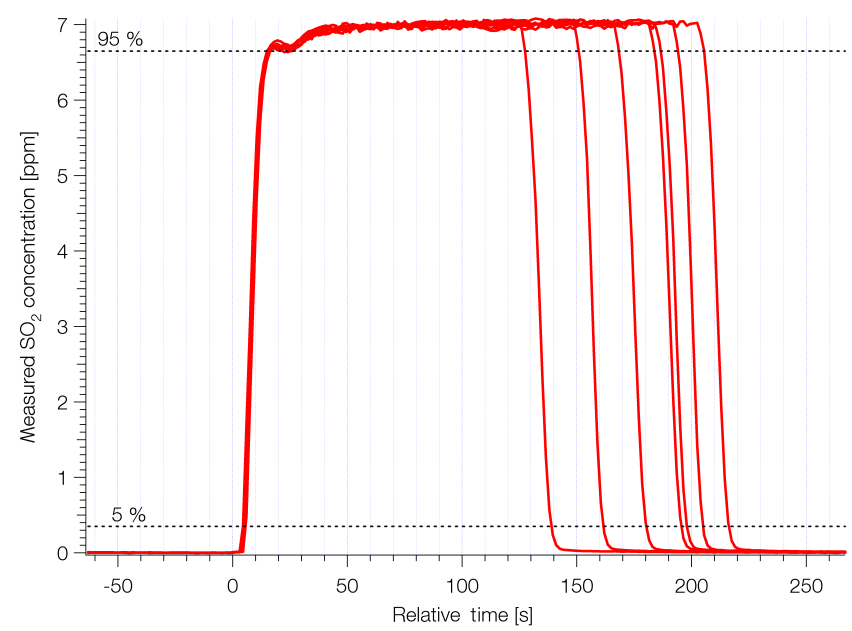

Figure 5. The trends in the $\mathrm{SO}_{2}$ concentration during five measurements of the zero and span test.

The noise behavior of the Q-MACS Trace system is characterized by means of an Allan variance analysis. In Fig. 6, the resulting Allan-Werle plot, as introduced by Werle as a powerful tool in the characterization of noise performance from laser-based sensors (Werle et al., 1993), is shown for a $3 \mathrm{~h}$ run of the sensor at a constant $\mathrm{SO}_{2}$ concentration. The plot shows a minimum detection limit of $4 \mathrm{ppb}(1 \sigma)$ with $70 \mathrm{~s}$ averaging.

\subsection{Operation under real conditions}

The developed sensor module was applied in a measurement campaign at Ancona Hospital in Italy (Ospedali Riuniti di Ancona). In this campaign, benchmarking was performed against an established FTIR-based measurement system: the Loccioni GIGAS 10M. The QCLAS sensor system was configured to support sulfur dioxide $\left(\mathrm{SO}_{2}\right)$ and methane $\left(\mathrm{CH}_{4}\right)$ as the target gases to be monitored in the compressed air supply line.

In Fig. 7, the results of a continuous measurement of $\mathrm{SO}_{2}$ by the FTIR and the QCL-based monitoring systems are shown. Also shown are the results for $\mathrm{CH}_{4}$ measured exclusively by the QCL absorption spectrometer. Methane is generally not regulated by the pharmacopeia, but as one of the most volatile compounds in oil released at temperatures of around $35^{\circ} \mathrm{C}$, it is one of the promising candidates for
Table 4. The data used to assess the zero and span drift of the QMACS Trace compact $\mathrm{SO}_{2}$ analyzer.

\begin{tabular}{lr}
\hline Component & Sensor $\mathrm{SO}_{2}(\mathrm{ppb})$ \\
\hline Pretest zero & $0.64 \pm 8.48$ \\
Pretest span & $7674.57 \pm 128.13$ \\
Posttest zero & $3.13 \pm 7.40$ \\
Posttest span & $7689.83 \pm 161.16$
\end{tabular}

Table 5. The results of the zero and span drift of the $\mathrm{SO}_{2}$ analyzer.

\begin{tabular}{lr}
\hline Component & Difference (ppb) \\
\hline Zero & 2.49 \\
Span & 15.26 \\
\hline Drift in \% of span & \\
\hline Zero & $0.04 \%$ \\
Span & $0.22 \%$ \\
\hline
\end{tabular}

oil contamination in the compressed air supply line. Because of the natural fluctuation of the $\mathrm{CH}_{4}$ concentration and the varying technologies used in hospital gas supplies, $\mathrm{CH}_{4}$ concentration measurements were analyzed with the aim of deriving unambiguous statements about the oil contamination of gas probes. While the QCL sensor system is connected to a bedside compressed air supply outlet, medical compressed air is generated through the mixing of pure gases. Therefore, impurities introduced by $\mathrm{SO}_{2}$ should be expected through leakages only. As the $\mathrm{SO}_{2}$ concentration in the ambient air is currently far below the detection limit of both systems, it is expected that no $\mathrm{SO}_{2}$ signal will be detectable. Furthermore, it should be noted that no specific procedures were applied to avoid water in the gas-handling system, which would react with the $\mathrm{SO}_{2}$ and reduce the concentration to be detected. Taking into account these considerations, the sensor signals are consistent as illustrated in the graph. An issue in the calibration data caused the observable irregularity in the $\mathrm{SO}_{2}$ concentration measured by the FTIR-based system. This illustrates the drawback of the multispeciescapable FTIR technique, as eminent cross sensitivities have to be respected carefully during the calibration process. Nevertheless, the false measurement is very close to the $\mathrm{SO}_{2}$ detection limit of the FTIR-based system. A reasonable correlation between the measurements of the different sensor types as well as good reproducibility can therefore be seen.

\section{Summary and outlook}

The test results, which are summarized in Table 6, confirm that the Q-MACS Trace analyzer provides a linear response over wide operating ranges. The compact prototype configu- 


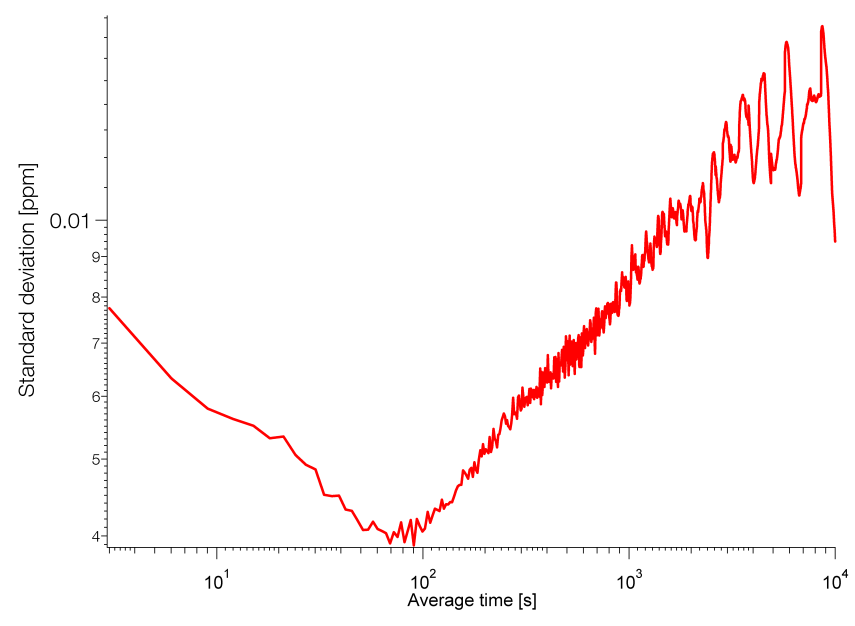

Figure 6. An Allan-Werle plot from 3 hours of continuous measurement by the Q-MACS $\mathrm{SO}_{2}$ sensor.

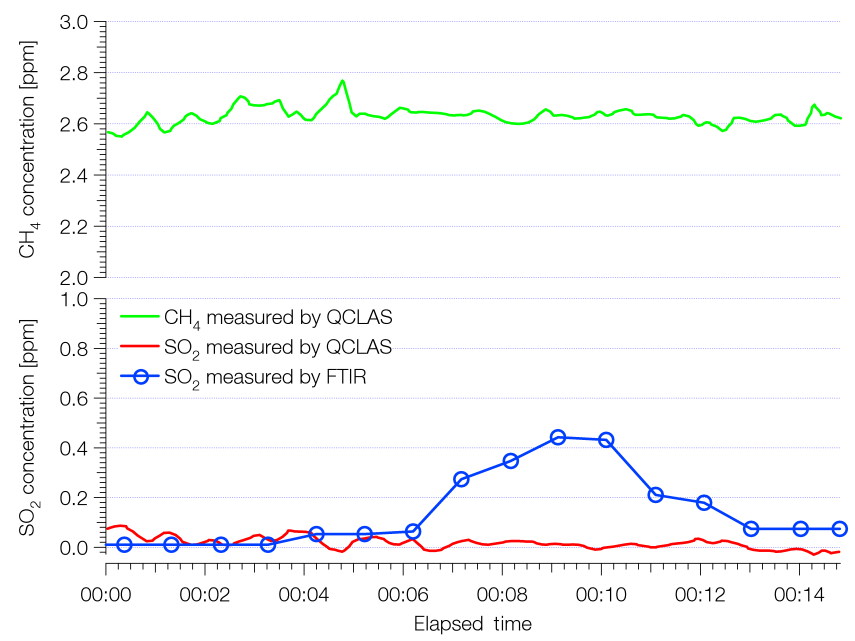

Figure 7. A comparison of the $\mathrm{SO}_{2}$ measurements by FTIR (blue) and QCLAS (red) as well as the $\mathrm{CH}_{4}$ measurements by QCLAS (green).

ration, as used in this preliminary study, provides very good results with respect to sensitivity, selectivity and stability.

The system is rugged and portable, and the necessary setup time is minimal. The fast sensor response times and measurement stability allowed for the verification testing to proceed smoothly. Its design incorporates a sample probe and a sample conditioning system, making it adaptable to a wide range of measurement applications.

Although the aim of $15 \mathrm{ppb}$ and below for the limit of detection was not achieved with this Q-MACS Trace compact configuration, the positive results show that it is possible to design a system that will fulfill this specification. Recently, long path cells with more than $150 \mathrm{~m}$ of optical path length have become available. Unfortunately, such a cell would not allow for the maintaining or even the further optimization
Table 6. The results from the performance analysis for the QMACS Trace $\mathrm{SO}_{2}$ sensor.

\begin{tabular}{lr}
\hline Specification & Value \\
\hline Response time & $14 \pm 1 \mathrm{~s}$ \\
Linearity & 1.048 \\
Detection limit & $<23 \mathrm{ppb}$ \\
\hline Drift & \\
\hline Zero & $<0.04 \%$ \\
Span & $<0.22 \%$ \\
\hline
\end{tabular}

of the compactness of the resulting sensor system. Moreover, the much higher volume of such a cell would lead to a significant and unwanted increase in the response time. A more practical approach is the selection of stronger absorption lines, which could be possible depending on the gas matrix to be analyzed in the specific application. Developments for the further optimization of the gas handling, e.g. through the integration of an active pressure control, and the extension of the analytical methods are currently in progress. Applying these drafted optimizations in a future configuration will allow for a decrease in the achievable detection limit by approximately a factor of $5 ; \mathrm{SO}_{2}$ concentrations below $5 \mathrm{ppb}$ could therefore be detected by a continuous monitoring system.

During an on-site measurement campaign, it was possible to convincingly demonstrate the applicability of QCLbased concentration monitoring solutions in hospital gas supply lines. With respect to the monitored $\mathrm{CH}_{4}$ concentration, it became obvious that this species is not sufficient as a unique marker for the determination of the oil contamination in gases. Therefore, efforts were made to incorporate additional target species into the QCL sensor system. In this context, it was possible to incorporate water vapor $\left(\mathrm{H}_{2} \mathrm{O}\right)$ as another target species of the sensor, which is relevant in the purity monitoring of the compressed air supply line as well. The measurements allow for the determination of the concentrations for selected impurities clearly below the threshold levels given by regulations such as those in the various pharmacopeia. This performance is mainly caused by the intrinsic, narrow laser-line width of the QCL and the capability to scan across the respective target spectrum with the highest spectral resolution, which makes it preferable under lower pressure conditions of a few hundred mbar as implemented in the solution developed within the project. This could be a disadvantage in the targeted application because it could result in the need for additional external gas-handling devices. This fact and the need for measuring even more species with a single sensor will be the basis for further developments to improve the device.

In pending development steps, the single-QCL source will be exchanged by a QCL array to address several molecular 
species in parallel while maintaining the compactness of the system. First introduced by Lee et al. (2009), QCL arrays will now make the step from the lab to the market as their technological relevance and applicability has already been demonstrated (Geiser et al., 2016).

Data availability. Research data are available upon request to the authors.

Competing interests. The authors declare that they have no conflict of interest.

Acknowledgements. This research was made possible thanks to funding from the European Union Seventh Framework Programme (FP7, 2007-2013) under grant agreement no. 17884 through the collaborative integrated project MIRIFISENS.

A special thank you also goes to the plasma diagnostic group at the Leibniz Institute for Plasma Science and Technology in Greifswald for the professional support during the final verification tests of the sensor configuration (http://www.inp-greifswald.de).

Edited by: J. Wöllenstein

Reviewed by: two anonymous referees

\section{References}

Anscombe, N.: Quantum leap, Electro Optics Issue, 210, 16-18, 2011.

Cornwall, J. and Robinson, B.: Out-of-sight killers - the ongoing problem with hospital gas lines, Journal of Health Design, 1, 25, doi:10.21853/JHD.2016.9, 2016.

Fischer, M., Tuzson, B., Hugi, A., Brönnimann, R., Kunz, A., Blaser, S., Rochat, M., Landry, O., Müller, A., and Emmenegger, L.: Intermittent operation of QC-lasers for midIR spectroscopy with low heat dissipation: tuning characteristics and driving electronics, Optics Express, 22, 7014-7027, ; doi:10.1364/OE.22.007014, 2014.

Geiser, P., Maisons, G., Dang, D., Brun, M., Carras, M., and Kasperse, P.: Multi-gas spectroscopy using continuous-wave quantum cascade laser arrays, Imaging and Applied Optics, OSA Technical Digest, doi:10.1364/LACSEA.2016.LTh3E.5, 2016.

Lee, B., Kansky, J., Goyal, A., Pflügl, C., Diehl, L., Belkin, M., Sanchez, A., and Capasso, F.: Beam combining of quantum cascade laser arrays, Optics Express, 17, 16216-16224, doi:10.1364/OE.17.016216, 2009.

Röpcke, J., Davies, P., Lang, N., Rousseau, A., and Welzel, S.: Applications of quantum cascade lasers in plasma diagnostics: a review, J. Phys. D Appl. Phys., 45, 10, doi:10.1088/00223727/45/42/423001, 2012.
Rothman, L., Gordon, I., Babikov, Y., Barbe, A., Benne, C., Bernath, P., Birk, M., Bizzocchi, L., Boudon, V., Brown, L., Campargue, A., Chance, K., Cohen, E., Coudert, L., Devi, V., Drouin, B., Fayt, A., Flaud, J.-M., Gamache, R., Harrison, J., Hartmann, J.-M., Hill, C., Hodges, J., Jacquemart, D., Jolly, A., Lamouroux, J., Le Roy, R., Li, G., Long, D., Lyulin, O., Mackie, C., Massie, S., Mikhailenko, S., Müller, H., Naumenko, O., Nikitin, A., Orphal, J., Perevalov, V., Perrin, A., Polovtseva, E., Richard, C., Smith, M., Starikova, E., Sung, K., Tashkun, S., Tennyson, J., Toon, G., Tyuterev, V., and Wagner, G.: The HITRAN2012 molecular spectroscopic database, J. Quant. Spectrosc. Ra., 130, 4-50, doi:10.1016/j.jqsrt.2013.07.002, 2013.

Weller, J., Merry, A., Warman, G., and Robinson, B.: Anaesthetists' management of oxygen pipeline failure: room for improvement, Anaesthesia, 62, 122-126, doi:10.1111/j.13652044.2006.04899.x, 2007.

Werle, P., Miicke, R., and Slemr, F.: The Limits of Signal Averaging in Atmospheric Trace-Gas Monitoring by Tunable Diode-Laser Absorption Spectroscopy (TDLAS), Appl. Phys. B, 57, 131-139, doi:10.1007/BF00425997, 1993. 